\title{
Fixed point analysis of limited range share per node wavelength conversion in asynchronous optical packet switching systems
}

\author{
Nail Akar • Ezhan Karasan • Carla Raffaelli
}

Received: 9 October 2008 / Accepted: 3 February 2009 / Published online: 25 February 2009

(C) Springer Science+Business Media, LLC 2009

\begin{abstract}
In this article, we study an asynchronous optical packet switch equipped with a number of wavelength converters shared per node. The wavelength converters can be full range or circular-type limited range. We use the algorithmic methods devised for Markov chains of block-tridiagonal type in addition to fixed-point iterations to approximately solve this relatively complex system. In our approach, we also take into account the finite number of fiber interfaces using the Engset traffic model rather than the usual Poisson traffic modeling. The proposed analytical method provides an accurate approximation for full range systems for relatively large number of interfaces and for circular-type limited range wavelength conversion systems for which the tuning range is relatively narrow.
\end{abstract}

Keywords Optical packet and burst switching - Limited range wavelength conversion · Markov chains · Fixed-point iterations

\section{Introduction}

Circuit switched optical networks are simple to build but they lack efficiency due to traffic burstiness. On the other hand, optical packet switched paradigms which aim at a bet-

\footnotetext{
N. Akar $(\bowtie) \cdot$ E. Karasan

Electrical and Electronics Engineering

Department, Bilkent University, Ankara, Turkey

e-mail: akar@ee.bilkent.edu.tr

E. Karasan

e-mail: ezhan@ee.bilkent.edu.tr

C. Raffaelli

DEIS - University of Bologna, Bologna, Italy

e-mail: carla.raffaelli@unibo.it
}

ter use of bandwidth have recently begun to mature. Two such paradigms are well-known: Optical Packet Switching (OPS) [1] and Optical Burst Switching (OBS) [2]. In this article, we analytically study the performance of an optical packet/burst switch using wavelength converters on a share per node (SPN) basis. Moreover, these converters can be full or circular-type limited range. As far as the current work is concerned, we do not differentiate between OPS and OBS since we deal with the forwarding but not the signaling plane and for the sake of simplicity, we will use the common term "(optical) packet" and "(optical) packet switching" to refer to a packet/burst and the data planes of OPS/OBS, respectively.

In synchronous (i.e., time-slotted) OPS, optical packets have fixed lengths and a need arises for costly synchronization equipment. Synchronous switching is known to be performance efficient due to the alignment of packet arrivals. On the other hand, optical packet lengths are variable in asynchronous (i.e., unslotted) optical switching and therefore packet arrivals need not be aligned. Although a debate currently exists on whether asynchronous or synchronous switching will be used in the future optical Internet, we believe that asynchronous packet switching is a more natural fit for supporting client networks carrying variable sized data packets, e.g., IP packets. In this article, we focus only on asynchronous OPS.

In OPS networks, contention arises when two or more incoming optical packets contend for the same output wavelength. Despite the existence of contention resolution mechanisms such as Fiber Delay Lines (FDL) and deflection routing [2], the simplest and most popular solution for contention resolution is to use wavelength conversion and in particular Tunable Wavelength Converters (TWC). In Full Wavelength Conversion (FWC), we have a dedicated TWC for each output wavelength channel. In Partial Wavelength Conversion (PWC), we have TWC sharing among a number 


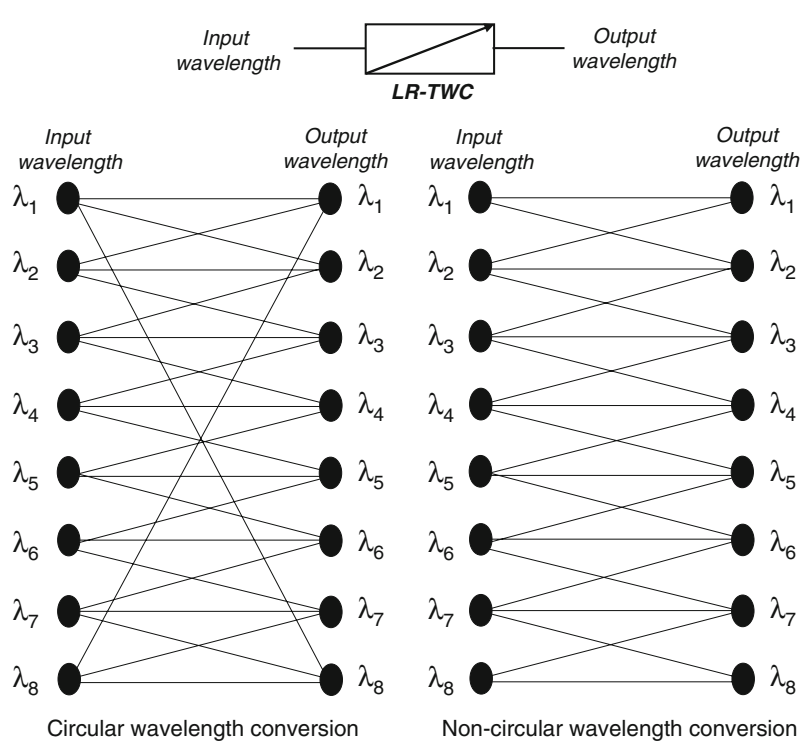

Fig. 1 Circular and non-circular conversion scheme depicted for $d=2$ for a WDM system with eight channels

of wavelength channels. Depending on how TWC sharing takes place, a number of architectures have been proposed for PWC. On one end, we have dedicated TWC banks for each output fiber line, called the Share Per Line (SPL) architecture [3]. On the other end, TWCs may be collected as a single converter pool for more efficient converter sharing across all fiber lines, which is referred to as the SPN architecture [3]. Besides, there are different architectures for TWCs which can be classified with respect to their tuning ranges. Full Range TWCs (FR-TWC) do not have tuning range limitations and they can convert an incoming wavelength to any other wavelength available in the system. In limited range wavelength conversion, a packet arriving on a wavelength can be converted to a fixed set of wavelengths above and below the original wavelength. Such converters are called Limited Range TWCs (LR-TWC) [4]. For LR-TWCs, conversion degree $d$ is defined as the total number of wavelengths available on both sides of the original wavelength for conversion purposes. LR-TWCs are also classified on the basis of the neighboring relationship for the wavelengths at the boundaries. In circular conversion, we assume the wavelengths are wrapped around to form a circle so that the wavelengths at the boundaries become neighbors. On the other hand, in non-circular-type limited range conversion, we do not allow wrap-around and the conversion ranges for wavelengths close to the boundaries are reduced in size. The difference between circular and non-circular wavelength conversion is presented in Fig. 1 that illustrates the adjacency set of each input wavelength in case of eight wavelength channels and $d=2$. In this article, we study only the circular conversion scheme.

The focus of the current article is on the performance analysis of a bufferless asynchronous optical packet switch

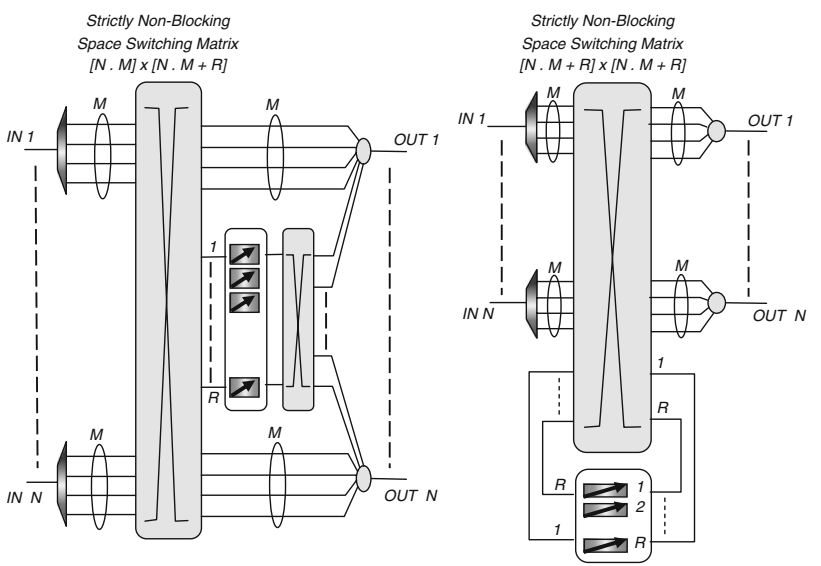

Fig. 2 Two switching node architectures with $N$ input and output fibers, $M$ wavelengths per fiber and limited number $R$ of LR-TWCs shared per node

employing SPN LR-TWCs (see Fig. 2 for two such architectures). In this scenario, the packet switching node is equipped with $N$ input/output fiber interfaces each carrying $M$ wavelengths. We also have $R$ LR-TWCs grouped together in a single bank so that an incoming packet can exploit any of the TWCs irrespective of the destination fiber line. The optical packet traffic is based on the Engset traffic model [5]. For each input wavelength channel (there are overall $K=M N$ input channels), the traffic is modeled with an $\mathrm{ON}-\mathrm{OFF}$ source. In this model, either the input channel is ON (an optical packet is being transmitted on that channel) or the input channel is OFF (the input channel is idle). We assume in this study that the ON and OFF times for each source are exponentially distributed with common means $1 / \mu$ and $1 / \lambda$, respectively. The offered load to the system is $\rho=\frac{\lambda}{(\lambda+\mu)}$. We also assume that each optical packet will be destined to one of the output fiber lines with probability $1 / N$. Therefore, the offered load for each output fiber line is $\rho$, i.e., symmetric loading. The generalization to more general traffic scenarios where loading on different output fiber lines is different, i.e., asymmetric loading, is left for future research. We call this traffic model a finite population traffic model since at any time there will at most be $K=N M$ packets destined to a particular output fiber line. This model is also known as the Engset model in the teletraffic literature and has been used for traffic modeling in optical packet switched networks [5]. The Engset model is different from infinite population models, e.g., Poisson model, where there may not be any upper limit on the maximum number of packets destined to a fiber line at a given time. In this respect, finite population models provide a better fit for switching systems with limited number of interfaces.

For SPL type converter sharing in asynchronous switching systems, the first exact algorithm is proposed in [6] that relies on the steady-state solution of a Markov chain and exploiting 
the block-tridiagonal structure of the underlying infinitesimal generator. Recently, a similar Continuous Time Markov Chain (CTMC)-based analysis is proposed in [7] for the same system and an approximate analytical method is proposed for the SPN converter sharing case using fixed-point iterations. On the other hand, the authors of [8] study an asynchronous SPN system and propose a new suite of methods to reduce the complexity of the multi-dimensional Markov chain. All studies above assume full range but shared wavelength conversion. Limited range conversion studies are rather rare. In [9], the authors provide an approximate method for SPL type converter sharing using LR-TWCs again using Markov chains and show that far conversion policies provide better performance when compared with random or near conversion policies for SPL type conversion. In [10], a product form solution is given for the special cases of $d=2$ and $d=4$, whereas an approximation technique is presented for more general scenarios for SPL type converter sharing. Studies on limited range wavelength conversion but for synchronous OPS systems are more mature [11]. Recently, a Markovian analysis is carried out in [12] for synchronous switching systems employing SPN type LR-TWCs. The contribution of the current article is 2 -fold. First, we use the idea of fixed-point iterations of [7] but for the relatively realistic on-off traffic model (as opposed to Poisson models) for studying SPN type converter sharing using FR-TWCs. While doing so, we benefit from the block-tridiagonal structure of the generators that arise using a technique similar to one introduced in [13]. Second, we propose novel approximations to the fullness probability of the tuning range as an enhancement of the approximation proposed in [9] as well as in [14] to deal with limited range conversion. We combine these two methods to provide a new approximative technique for the performance analysis of switching systems using full or circular-type limited range SPN wavelength conversion.

The outline of the article is as follows. The approximate analytical method is presented in Sect. 2 to calculate packet blocking probabilities in the switching system of interest. Numerical results are presented in Sect. 3. We conclude in the final section.

\section{Analytical method}

Let us first concentrate on a single output fiber (tagged fiber) which consists of $M$ wavelength channels. Recall that the other fibers are statistically equivalent and the stochastic analysis of the tagged fiber will be sufficient for analyzing the entire system. In this case, an incoming optical packet destined to the tagged fiber (with probability $1 / N$ ) is forwarded without conversion if its incoming wavelength is idle on the outgoing link. If the incoming wavelength is occupied then there are two possibilities: if there is an idle wavelength in the tuning range then the packet will be directed to the converter pool or otherwise the packet will be blocked. In the former case, if all the converters are in use then the packet will again be blocked otherwise the packet will be directed to the destination fiber using one of the free converters and one of the available wavelengths in the tuning range. In the random (far) conversion policy, an idle wavelength (farthest idle wavelength) from the tuning range is selected as the outgoing wavelength. Far conversion policies are known to yield better performance than random conversion policies as explained in [9].

In this model, there are two interacting processes; one of them is the tagged fiber process and the other one being the wavelength conversion process. The tagged fiber process keeps track of the channel occupancy of the tagged fiber, whereas the wavelength conversion process keeps track of the occupancy of the conversion pool. The state space required to keep track of all the output fibers in the system as well as the converter pool would be enormously large making it impossible to solve the arising Markov chain. This problem is known as the curse of dimensionality in Markov chains. We therefore need to make the following two assumptions to be able to approximately solve this very complex problem.

- Assumption A: We assume the tagged fiber process is impacted only by the wavelength conversion process via the blocking probability $P_{\text {blocked }}^{\text {conv }}$ which is defined as the probability of blocking due to the lack of a converter in the conversion pool given that the optical packet is directed to the converter pool. On the other hand, the wavelength conversion process is impacted by the tagged fiber process only through the probability $P_{\text {directed }}$ which is defined as the probability that an incoming packet directed to the tagged fiber is also directed to the converter pool.

- Assumption B: Second, in the actual system, there can at most be $N$ optical packets in the ON state for a given wavelength $j$. Instead, we assume in our simplified model that we have $K=M N$ input channels for which an optical packet on a given channel can belong to wavelength $j$ with probability $1 / M$. This simplified model frees us from the burden of keeping track of individual input wavelengths.

With these two assumptions in place, let us first concentrate on the tagged fiber process. For mathematical analysis, let $i(t)$ and $j(t)$ denote the number of wavelength channels that are in use on the tagged fiber and the number of input wavelength channels that are in the ON state, respectively, at time $t$. Under the two assumptions described above, the tagged fiber process $X(t)=\{(i(t), j(t)): t \geq 0\}$ can be shown to be a Markov process on the state space $S=\{(i, j)$ : $0 \leq j \leq K, 0 \leq i \leq \min (M, j)\}$. To see this, let us assume that the process is in some state $(i, j)$ at time $t$. If a new packet arrives in the interval $(t, t+\Delta t)$ which occurs with 
probability $(K-j) \lambda \Delta t+O(\Delta t)$ (i.e., $\lim _{\Delta t \rightarrow 0} O(\Delta t) / \Delta t=$ $0)$ [15], then the packet will be destined to the tagged fiber with probability $1 / N$. Otherwise, the packet is destined to another fiber and the Markov chain governing the tagged fiber will jump to state $(i, j+1)$. When the arriving packet is destined to the tagged fiber, it will require conversion with probability $i / M$ (from Assumption B); otherwise the packet will be directed to the tagged fiber and the Markov chain will jump to state $(i+1, j+1)$ (or will be blocked when $i(t)=M$ and the visited state will be $(i, j+1))$. When the packet requires conversion, we check the fullness probability of the tuning range denoted by $P_{f u l l}(i, d, M)$ as a function of $i, d$, and $M$ for an incoming packet finding $i$ channels occupied (including the original wavelength) and requiring conversion. However, it is hard to derive this quantity for which we propose two approximations (approximations A and B); the first one based on [9]. In approximation $\mathrm{A}$, the conversion range is not the actual $\pm d / 2$ neighborhood of the incoming wavelength but is instead taken as a set of arbitrarily selected $d$ wavelengths at each time conversion is to take place. This approximative model captures the impact of degree of conversion, but does not reflect the clustering effect mentioned in [9]. To summarize the clustering effect, the probability of a packet to find its conversion range fully occupied is larger than the full occupancy probability of an arbitrarily selected set of $d$ wavelengths other than the incoming wavelength. Equivalently, there is a positive spatial correlation between the status of two neighboring wavelengths and consequently occupied wavelengths tend to cluster in time as opposed to the case of FR-TWCs. We call this effect the clustering effect which obviously has a detrimental impact on blocking performance.

We now have the following Approximation A:

$$
P_{\text {full }}^{A}(i, d, M)= \begin{cases}\frac{i-1}{M-1} \frac{i-2}{M-2} \cdots \frac{i-d}{M-d} & \text { if } i \geq d+1 \\ 0 & \text { if } i<d+1 .\end{cases}
$$

On the other hand, Approximation B takes into account the clustering effect. When a packet $k$ coming on wavelength $j$ requires conversion then wavelength $j$ on the tagged fiber is occupied by an ongoing packet say $l$. There are two possibilities: either this ongoing packet $l$ is riding on its original wavelength or this packet itself has been converted with a probability $P_{\text {converted }}$, which is defined as the probability that a successfully transmitted packet required conversion. In the latter case, another wavelength in the tuning range should also be occupied at the arrival epoch of packet $l$. However, it is not difficult to show that this particular wavelength is still occupied at the arrival epoch of packet $k$ with probability 0.5 . To see this, the time between the arrival of packets $k$ and $l$ is exponentially distributed with parameter $\mu$ [15]. On the other hand, the length of packet $l$ is also exponentially distributed with parameter $\mu$. The probability of the latter being larger than the former is obviously 0.5 . Therefore, with probability
$0.5 P_{\text {converted }}$ a wavelength in the tuning range should also be occupied at the arrival epoch of packet $k$ which then forms the basis for the improved approximation B:

$$
\begin{aligned}
P_{\text {full }}^{B}(i, d, M)= & \left(1-0.5 P_{\text {converted }}\right) P_{\text {full }}^{A}(i, d, M) \\
& +0.5 P_{\text {converted }} P_{\text {full }}^{A}(i-1, d-1, M-1)
\end{aligned}
$$

The full-range wavelength conversion case can be obtained by setting $d=M$ which then yields a zero probability of tuning range fullness. If the tuning range is not full then the packet is directed to the converter pool which comprises $R$ converters and the packet will either be blocked due to the lack of converters with probability $P_{\text {blocked }}^{\text {conv }}$ and the visited state will be $(i+1, j)$ or the packet will use one of the free converters so as to be directed to the tagged fiber and the Markov chain will jump to state $(i+1, j+1)$. If a packet departure occurs in the interval $(t, t+\Delta t)$ which occurs with probability j $\mu \Delta t+O(\Delta t)$, then the Markov chain will jump to state $(i-1, j-1)$ with probability $1 / N$ and to $(i-1, j)$ otherwise. It is thus clear that the process $X(t)$ is a Continuous Time Markov Chain (CTMC) and the infinitesimal generator of the CTMC possesses a block-tridiagonal form if the states are properly enumerated as

$$
\begin{aligned}
& S=\{\underbrace{(0,0)}_{\text {level } 0}, \underbrace{(0,1),(1,1)}_{\text {level } 1}, \\
& \underbrace{(0,2),(1,2),(2,2)}_{\text {level } 2}, \ldots, \underbrace{(0, K), \ldots,(M, K)}_{\text {level } K}\} \text {. }
\end{aligned}
$$

A numerically stable and efficient solution procedure, the so-called block-tridiagonal LU factorization algorithm can then be used to find the stationary solution of the underlying CTMC while taking advantage of the block-tridiagonal structure of the generator [16, pp. 174-175]. The complexity of the block-tridiagonal LU factorization algorithm is $O\left(K M^{3}\right)$. This is in contrast with the $O\left(K^{3} M^{3}\right)$ computational complexity governing the brute force approach. Therefore switching systems with large number of interfaces are not beyond reach as will be shown throughout the numerical examples. We derive two quantities using this model; one of them is $P_{\text {directed }}$ which denotes the probability than an incoming packet directed to the tagged fiber is also directed to the converter pool and the other one is $P_{\text {blocked }}$, the overall blocking probability. For this derivation, let $x$ be the steadystate vector and $x(i, j)$ be the steady-state probability of finding the Markov process at state $(i, j)$ at an arbitrary epoch. We first write

$$
\begin{aligned}
P_{\text {directed }}= & \frac{1}{\Delta} \sum_{j=1}^{K} \sum_{i=1}^{M-1} x(i, j)(K-j)(i / M) \\
& \left(1-P_{\text {full }}(i, d, M)\right),
\end{aligned}
$$


where $\Delta=\frac{K \mu}{\lambda+\mu}$ and we write $P_{\text {blocked }}=\frac{1}{\Delta}$ times the following quantity:

$$
\begin{aligned}
& \sum_{j=1}^{K} \sum_{i=1}^{M-1} x(i, j)(K-j)(i / M)\left(1-P_{\text {full }}(i, d, M)\right) P_{\text {blocked }}^{\text {conv }} \\
& \quad+\sum_{j=1}^{K} \sum_{i=1}^{M-1} x(i, j)(K-j)(i / M) P_{\text {full }}(i, d, M) \\
& \quad+\sum_{j=1}^{K}(K-j) x(M, j),
\end{aligned}
$$

where we substitute $P_{\text {full }}^{A}(i, d, M), P_{\text {full }}^{B}(i, d, M)$, and 0 , in place of $P_{f u l l}(i, d, M)$ in Eqs. 3 and 4, for approximations A and B for limited range systems and the full range system, respectively. At this point, we can calculate the probabilities $P_{\text {directed }}$ and $P_{\text {blocked }}$ upon a-priori information about $P_{\text {converted }}$ and $P_{\text {blocked }}^{\text {conv }}$. However, note that

$$
P_{\text {converted }}=P_{\text {directed }}\left(1-P_{\text {blocked }}^{\text {conv }}\right) /\left(1-P_{\text {blocked }}\right),
$$

which would require the solution of a fixed-point equation. Moreover, the latter quantity $P_{\text {blocked }}^{c o n v}$ also needs to be calculated. We will now show that $P_{\text {blocked }}^{\text {conv }}$ can be calculated using $P_{\text {directed }}$ which will lead us to a fixed-point iteration. To see this, first note that $P_{\text {directed }}$ is also the probability that an arriving packet is directed to the converter pool due to symmetry among fibers. Let us now concentrate on the following problem which consists of $K$ on-off sources with each packet (corresponding to an ON time) directed to the pool of $R$ converters with probability $P_{\text {directed }}$. The blocking probability in this new system gives us $P_{\text {blocked }}^{\text {conv }}$. For this system, let $i(t)$ and $j(t)$ denote the number of TWCs that are in use and the number of sources that are in the $\mathrm{ON}$ state, respectively. The process $Y(t)=\{(i(t), j(t)): t \geq 0\}$ is then a Markov process on the state space $S=\{(i, j): 0 \leq j \leq K, 0 \leq i \leq$ $\min (R, j)\}$. To show this, let us assume that the process is in some state $(i, j)$ at time $t$. If a new packet arrives which occurs with rate $(K-j) \lambda$ then the packet will be directed to the converter pool with probability $P_{\text {directed }}$. The packet will be admitted into the system if $i<R$ and the Markov chain will jump to state $(i+1, j+1)$ or will be blocked when $i(t)=R$ and the visited state will be $(i, j+1)$. If a packet departure occurs (with rate $j \mu$ ) then the Markov chain will jump to state $(i-1, j-1)$ with probability $\frac{i}{j}$ or to $(i, j-1)$ with probability $1-\frac{i}{j}$. This shows that the underlying system is Markov and this system again has a block-tridiagonal generator. Solving for the steady-state probabilities of finding the system in state $(i, j)$ denoted by $y(i, j)$ using the abovementioned block-tridiagonal LU factorization algorithm, we finally have
Table 1 Iterative algorithm to calculate the overall blocking probability Plocked

1. First start with arbitrary initial probabilities, say $P_{\text {blocked }}^{\text {conv }}=P_{\text {directed }}=P_{\text {blocked }}=0$

2. Given $P_{\text {blocked }}^{\text {conv }}, P_{\text {directed }}$, and $P_{\text {blocked }}$, first calculate $P_{\text {converted }}$ according to (5) and then construct and solve the Markov chain governing the tagged fiber process $X(t)$ and obtain the steady-state probabilities $x(i, j)$

3. $\quad$ Find $P_{\text {directed }}$ using (3)

4. Write $P_{\text {blocked }}$ through (4) which gives us an approximation for the blocking probability. If the normalized difference between the two successive values of $P_{\text {blocked }}$ is less than an a-priori given parameter $\varepsilon$, then exit the loop

5. Given $P_{\text {directed }}$, construct and solve the Markov chain governing the conversion process $Y(t)$ and obtain the steady-state probabilities $y(i, j)$

6. Find $P_{\text {blocked }}^{\text {conv }}$ through (6)

7. Go to step 2

$P_{\text {blocked }}^{\text {conv }}=\frac{1}{\Delta}\left(\sum_{j=R}^{K} y(R, j)(K-j)\right)$.

Note that the complexity of the block-tridiagonal LU factorization algorithm used for the converter process is $O\left(K R^{3}\right)$ and this may be formidable especially when $R \rightarrow K$. The whole iterative procedure is summarized in Table 1.

\section{Numerical examples}

For all numerical examples to follow, we take $\mu=1$. In the first numerical example, we study the accuracy of the proposed analytical method for full-range wavelength conversion case. For this purpose, we first introduce a wavelength conversion percentage ratio parameter $r=100 \frac{R}{N M}$. We use the algorithmic procedure of Table 1 for fixed $M=8$ and for varying $N$, for two different values of $\rho=0.3,0.7$, and for three different values of $r=6.25,25$, and $50 \%$. Note that we substitute zero in place of $P_{\text {full }}(i, d, M)$ since we are

\begin{tabular}{|c|c|c|c|c|c|c|}
\hline \multirow[t]{2}{*}{$N$} & \multicolumn{3}{|l|}{$\rho=0.3$} & \multicolumn{3}{|l|}{$\rho=0.7$} \\
\hline & $r=6.25 \%$ & $r=25 \%$ & $r=50 \%$ & $r=6.25 \%$ & $r=25 \%$ & $r=50 \%$ \\
\hline 2 & 6 & 5 & 3 & 6 & 7 & 6 \\
\hline 4 & 6 & 4 & 2 & 6 & 7 & 5 \\
\hline 8 & 7 & 3 & 2 & 6 & 7 & 4 \\
\hline 12 & 7 & 3 & 2 & 6 & 7 & 3 \\
\hline 16 & 7 & 2 & 2 & 6 & 7 & 3 \\
\hline 24 & 7 & 2 & 2 & 6 & 7 & 3 \\
\hline 32 & 7 & 2 & 2 & 6 & 7 & 2 \\
\hline 48 & 7 & 2 & 2 & 6 & 7 & 2 \\
\hline 64 & 7 & 2 & 2 & 6 & 7 & 2 \\
\hline
\end{tabular}

Table 2 Number of iterations required for convergence for the proposed algorithm for different values of $N, \rho$, and $r$, with $M=8$ and the stopping criterion parameter $\varepsilon$ set to $10^{-4}$ 


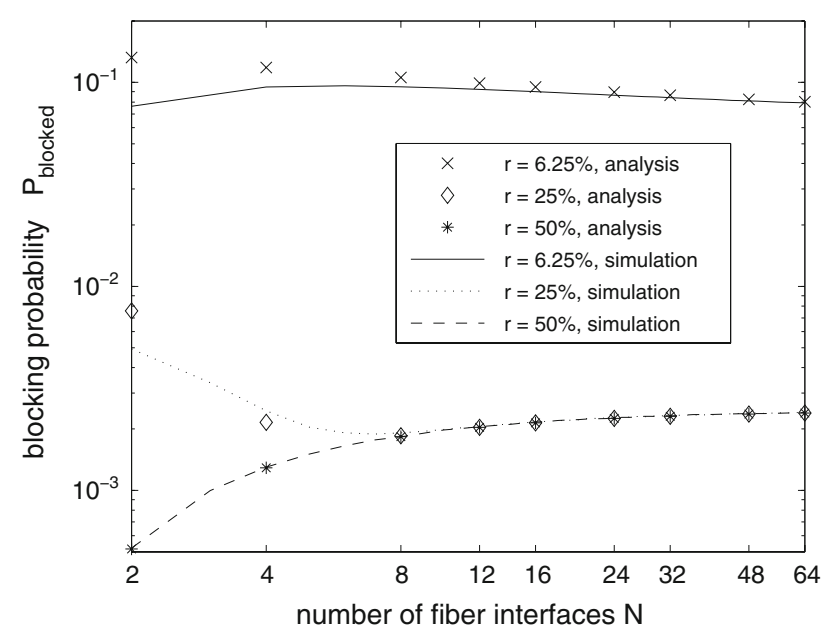

Fig. 3 Blocking probability $P_{\text {blocked }}$ as a function of the number of interfaces $N$ for an 8-wavelength system with $\rho=0.3$ and for three values of $r=6.25,25$, and $50 \%$

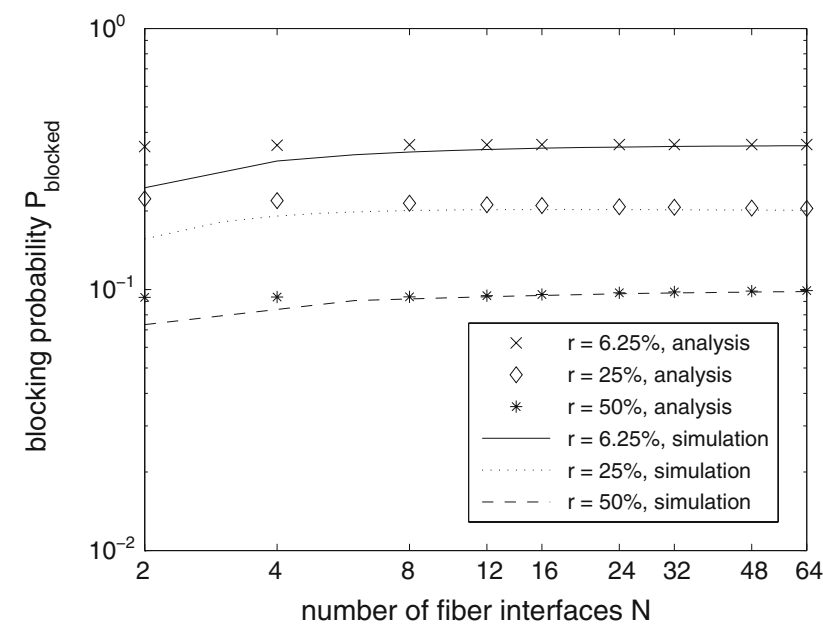

Fig. 4 Blocking probability $P_{\text {blocked }}$ as a function of the number of interfaces $N$ for an 8-wavelength system with $\rho=0.7$ and for three values of $r=6.25,25$, and $50 \%$

dealing with FR-TWCs. We take the stopping criterion parameter $\varepsilon=10^{-4}$. The results are given in Figs. 3 and 4 .

We first observe that the analytical approach produces very accurate results especially when $N \geq 8$ since the independence assumption between the tagged fiber process and the conversion process is most justified when $N$ is relatively large. Second, we observe that there are two effects counteracting each other when we vary $N$; the first one is when we have full conversion, i.e., $r \rightarrow 100 \%$, the blocking probability increases with increasing $N$ since with more interfaces the output contention probability increases as explained in [17]. However, when we have PWC, we have better sharing of converter resources when $N$ increases due to economy of scale which leads to reduced blocking probabilities. These counteracting effects are illustrated in Fig. 3 where the blocking

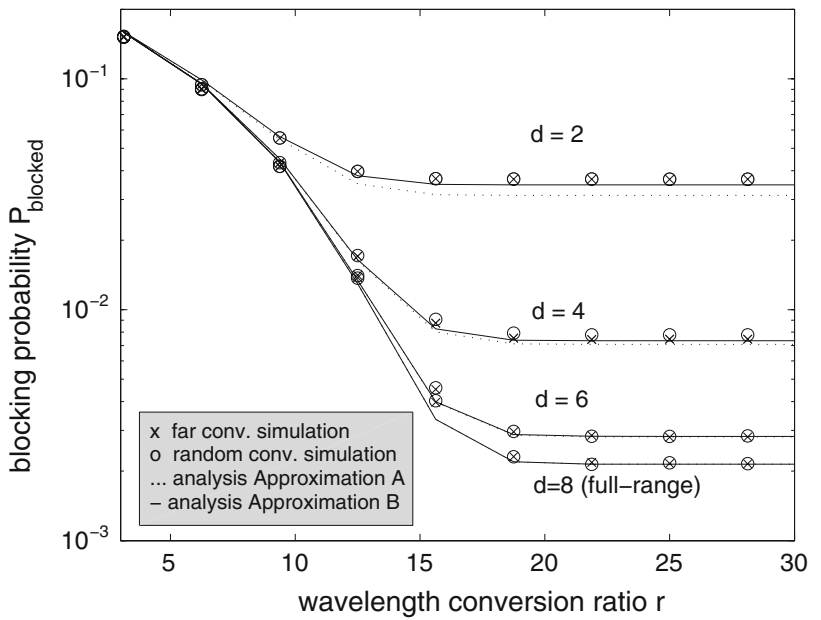

Fig. 5 Blocking probability as a function of the wavelength conversion ratio $r$ for an 8-wavelength system with $N=16, \rho=0.3$, and for different values of the degree parameter $d$

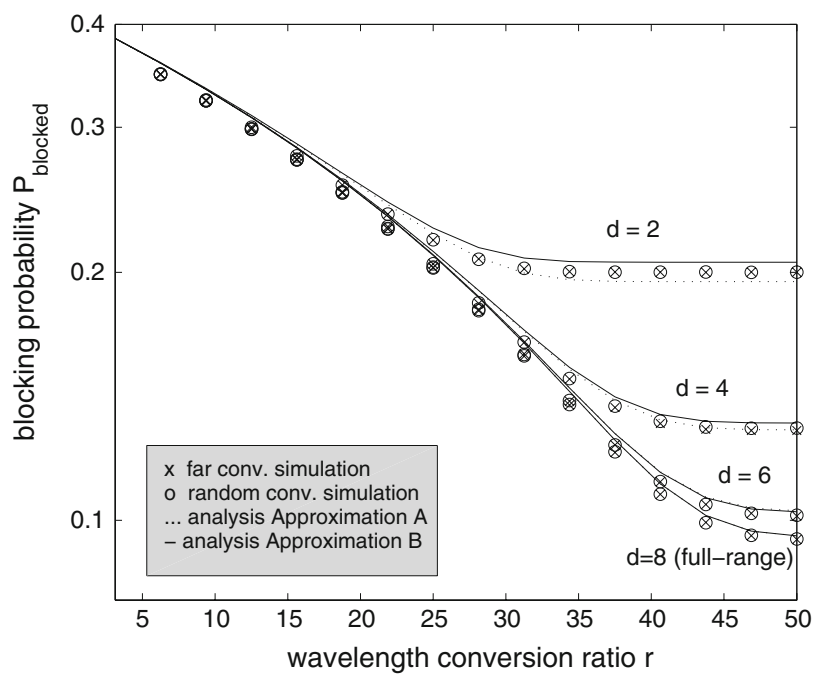

Fig. 6 Blocking probability as a function of the wavelength conversion ratio $r$ for an 8-wavelength system with $N=16, \rho=0.7$, and for different values of the degree parameter $d$

probability decreases (increases) for low (high) conversion ratios and we observe both effects for a moderate conversion ratio when we have light load, i.e., $\rho=0.3$. In Fig. 4, which is for a higher load ( $\rho=0.7)$, we observe only the former effect and the blocking rate strictly increases with increasing $N$ for different conversion ratios. For the same numerical example, the number of required iterations is presented in Table 2. It is clear that the algorithm converges quite rapidly although we do not have a formal proof of convergence. Convergence rates of the algorithm appear to be higher for increased conversion ratios and lighter loads.

In the second numerical example, we study the accuracy of the proposed analytical method for limited range wavelength conversion. We run the algorithmic procedure with the two Approximations A and B given in Table 1 for $M=8$ 


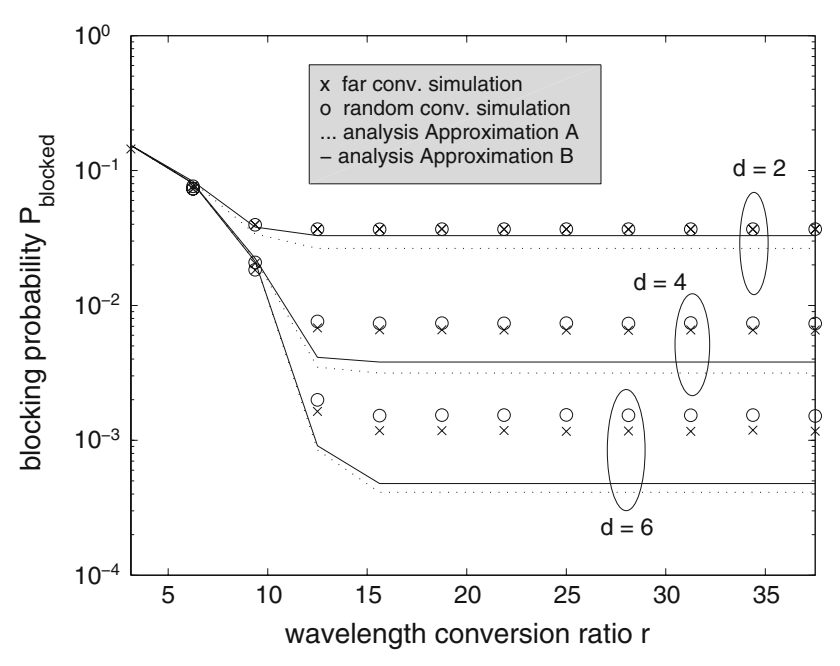

Fig. 7 Blocking probability as a function of the wavelength conversion ratio $r$ for an 32-wavelength system with $N=16, \rho=0.3$, and for different values of the degree parameter $d$

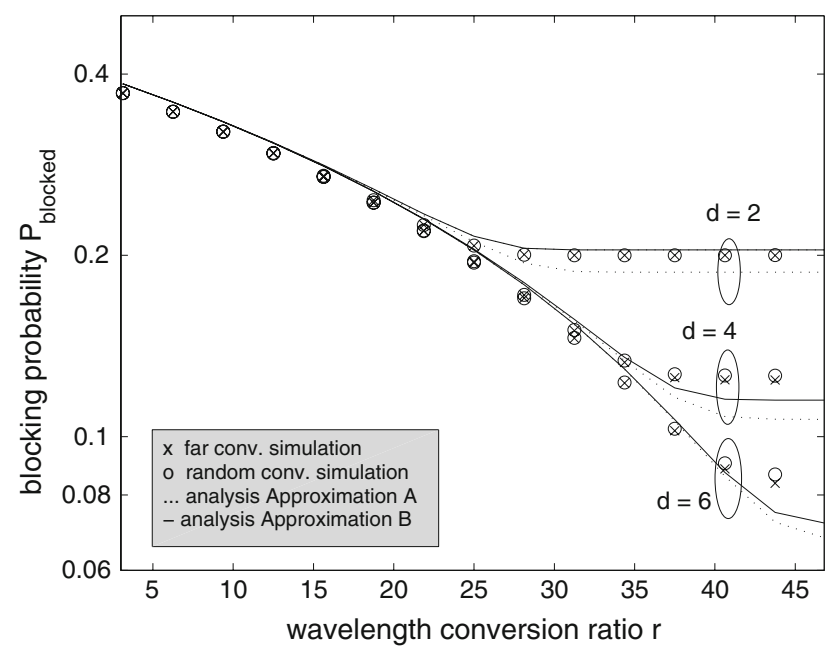

Fig. 8 Blocking probability as a function of the wavelength conversion ratio $r$ for an 32-wavelength system with $N=16, \rho=0.7$, and for different values of the degree parameter $d$

and $\rho=0.3,0.7$, but for varying $d$ and $r$ parameters with the total number of algorithm iterations set to eight. The results are depicted in Figs. 5 and 6. We repeat the same experiment above but with $M=32$ whose results are presented in Figs. 7 and 8 . We now summarize our findings below:

1. The far conversion policy outperforms the random conversion policy where the gain in using far conversion is more significant with increased $M$ whereas for $M=8$, the difference between the two policies is marginal. Note that when $d=2$, the two policies are equivalent by definition and they deviate from each other when $d>2$. On the other hand, when $d \rightarrow M$, we approach the scenario of full range conversion for which again far and random conversion policies are equivalent. Both analytical methods (approximations A and B) appear to provide a better approximation to far conversion than random conversion.

2. We show that the Approximation B generally improves upon Approximation A by taking the clustering effect into consideration. Specifically, this improved approximation provides very accurate results for $d=2$ and for different values of $M, \rho$, and $r$. However, the accuracy of this approximation drops for $d>2$ especially for increased number of wavelengths $M$ which leads us to believe that refined approximations are needed for the case $d>2$.

3. When load is relatively low, i.e., $\rho=0.3$, the blocking probabilities saturate at around $r=20 \%$ for $M=8$ and for $r=15 \%$ for $M=32$ irrespective of the values of the degree parameter $d$. This observation leads us to believe that the use of FWC for SPN systems may not be as necessary especially for low to moderate loads and relatively large number of wavelengths per fiber. We also observe that this saturation behavior slows down with increased load, i.e., $\rho=0.7$.

\section{Conclusions}

In this article, we study an asynchronous optical switch with SPN circular-type limited range wavelength converters. The traffic is modeled as a superposition of identically distributed on-off sources. We use fixed-point iterations in conjunction with block-tridiagonal LU factorizations to obtain an approximate solution to the blocking probabilities. The proposed technique for the full range conversion case is accurate with increased number of fiber interfaces. Moreover, the proposed approximations appear to be very accurate for limited range conversion case for wide range of problem parameters for the particular case of $d=2$ and they start to deviate from the simulation results with increased $d$. The gap between the simulations and the proposed approximation is more elaborate with increased number of channels $M$. The proposed approximations can be used for converter dimensioning purposes.

For future work, we plan on working on enhanced approximations that will give more accurate results for $d>2$. Moreover, methods with lesser computational complexity are required to solve problems with large size since the $O\left(K R^{3}\right)$ computational complexity of the algorithm proposed for the wavelength conversion process may become substantial when $R \rightarrow K$.

Acknowledgments The work described in this article was carried out with the support of the BONE-project (Building the Future Optical Network in Europe), a Network of Excellence funded by the European Commission through the 7th ICT-Framework Programme, and in part by The Science and Research Council of Turkey (Tübitak) under grant no. EEEAG-106E046. 


\section{References}

[1] Gambini, P. et al.: Transparent optical packet switching: network architecture and demonstrators in the KEOPS project. IEEE J. Select Areas Commun. 16, 1245-1259 (1998)

[2] Qiao, C., Yoo, M.: Optical burst switching (OBS)—a new paradigm for an optical Internet. J. High Speed Netw. (JHSN) 8, 6984 (1999)

[3] Eramo, V., Listanti, M., Pacifici, P.: A comparison study on the wavelength converters number needed in synchronous and asynchronous all-optical switching architectures. J. Lightw. Technol. 21, 340-355 (2003)

[4] Puttasubbappa, V., Perros, H.: An approximate queueing model for limited-range wavelength conversion in an OBS switch. In: Boutaba, R. et al. (eds.) Networking, LNCS 3426, pp. 697-708. Ontario, Canada (2005)

[5] Overby, H.: Performance modelling of optical packet switched networks with the Engset traffic model. Opt. Express 13, 16851695 (2005)

[6] Akar, N., Karasan, E.: Exact calculation of blocking probabilities for bufferless optical burst switched links with partial wavelength conversion. In: 1st Conference on Broadband Networks (BROADNETS'04), Optical Networking Symposium, pp. 110-117 (2004)

[7] Mingwu, Y., Zengji, L., Aijun, W.: Accurate and approximate evaluations of asynchronous tunable-wavelength-converter sharing schemes in optical burst-switched networks. J. Lightw. Technol. 23, 2807-2815 (2005)

[8] Li, H., Thng, I.L.-J.: Performance analysis of limited number of wavelength converters by share per node in optical switching network. Comput. Netw. 51, 671-682 (2007)

[9] Dogan, K., Gunalay, Y., Akar, N.: Comparative study of limited range wavelength conversion policies for asynchronous optical packet switching. J. Opt. Netw. 6, 134-145 (2007)

[10] Puttasubbappa, V., Perros, H.: Performance analysis of limitedrange wavelength conversion in an OBS switch. Telecommun. Syst. J. 31, 227-246 (2006)

[11] Eramo, V., Listanti, M., Spaziani, M.: Resources sharing in optical packet switches with limited-range wavelength converters. J. Lightw. Technol. 23, 671-687 (2005)

[12] Savi, M., Raffaelli, C., Akar, N., Karasan, E.: Traffic analysis of synchronous buffer-less optical switch with shared limited range wavelength converters. IEEE High Performance Switching and Routing (HPSR), New York (2007)

[13] Akar, N., Karasan, E., Dogan, K.: Wavelength converter sharing in asynchronous optical packet/burst switching: an exact blocking analysis for Markovian arrivals. IEEE J. Select Areas Commun. 24, 69-80 (2006)

[14] Akar, N., Karasan, E., Muretto, G., Raffaelli, C.: Performance analysis of an optical packet switch employing full/limited range share per node wavelength conversion. In: IEEE GLOBECOM, Washington, DC, pp. 2369-2373 (2007)

[15] Kleinrock, L.: Queuing Systems, Vol. 1 Theory. John Wiley, New York (1975)

[16] Golub, G.H., van Loan, C.F.: Matrix Computations. 3rd edn. The Johns Hopkins University Press, Baltimore, Maryland (1996)

[17] Akar, N., Gunalay, Y.: Stochastic analysis of finite population bufferless multiplexing in optical packet/burst switching systems. IEICE Trans. Commun. E E90-B(2), 342-345 (2007)
Author Biographies

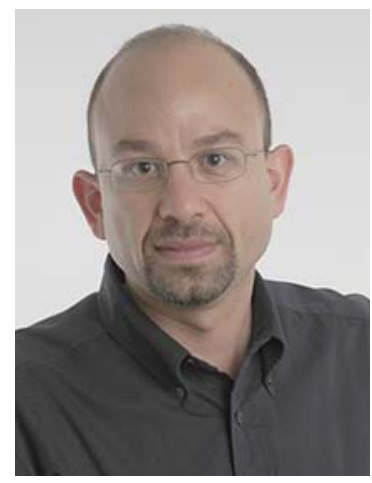

Nail Akar received the B.S. degree from Middle East Technical University, Turkey, in 1987 and M.S. and $\mathrm{Ph} . \mathrm{D}$. degrees from Bilkent University, Turkey, in 1989 and 1994, respectively, all in electrical and electronics engineering. From 1994 to 1996, he was a visiting scholar and a visiting assistant professor in the Computer Science Telecommunications program at the University of Missouri - Kansas City. He joined the Technology Planning and Integration group at Long Distance Division, Sprint,

Overland Park, Kansas, in 1996, where he held a senior member of technical staff position from 1999 to 2000. Since 2000, he has been with Bilkent University, currently as an associate professor. He is actively involved in European Commission FP7 projects BONE and Wimagic. This year, Nail Akar has been one of the technical program co-chairs of the 2008 IEEE IP Operations and Management Workshop and the 2008 ICCCN Track on QoS Control and Traffic Modeling. His current research interests include performance analysis of computer and communication networks, optical networks, queueing systems, traffic control and resource allocation.

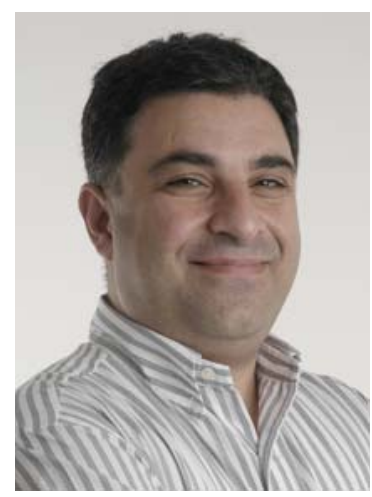

Ezhan Karasan received the B.S. degree from Middle East Technical University, Ankara, Turkey, M.S. degree from Bilkent University, Ankara, Turkey, and Ph.D. degree from Rutgers University, Piscataway, New Jersey, USA, all in electrical engineering, in 1987, 1990, and 1995, respectively. During 1995-1996, he was a postdoctorate researcher at Bell Labs, Holmdel, New Jersey, USA. From 1996 to 1998 , he was a Senior Technical Staff Member in the Light-wave Networks Research Department at AT\&T Labs-Research, Red Bank, New Jersey, USA. $\mathrm{He}$ has been with the Department of Electrical and Electronics Engineering at Bilkent University since 1998, where he is currently an associate professor. During 1995-1998, he worked in the Long Distance Architecture task of the Multiwavelength Optical Networking Project (MONET), sponsored by DARPA. Dr. Karasan is a member of the Editorial Board of Optical Switching and Networking journal. He is the recipient of 2004 Young Scientist Award from Turkish Scientific and Technical Research Council (TUBITAK), 2005 Young Scientist Award from Mustafa Parlar Foundation and Career Grant from TUBITAK in 2004. Dr. Karasan received a fellowship from NATO Science Scholarship Program for overseas studies in 1991-1994. Dr. Karasan is currently the Bilkent team leader of the FP7-IST Network of Excellence (NoE) project BONE. His current research interests are in the application of optimization and performance analysis tools for the design, engineering and analysis of optical networks and wireless ad hoc/sensor networks. 


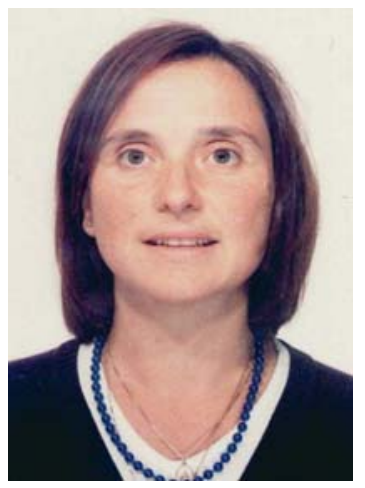

Carla Raffaelli is an associate professor in switching systems and telecommunication networks at the University of Bologna. She received the M.S. and the Ph.D degrees in electrical engineering and computer science from the University of Bologna, Italy, in 1985 and 1990, respectively. Since 1985 she has been with the Department of Electronics, Computer Science and Systems of the University of Bologna, Italy, where she became a Research Associate in 1990. Her research interests include performance analysis of telecommunication networks, switching architectures, protocols and broadband communication. Since 1993 she participated in European funded projects on optical packet-switched networks, the RACEATMOS, the ACTS-KEOPS and the IST-DAVID projects. She was active in the EU funded e-photon/One network of excellence and, at present, in its follow-up, BONE. She also participated in many national research projects on multi-service telecommunication networks. She is the author of many technical papers on broadband switching and network modeling and acts as a reviewer for top international conferences and journals. She is author or co-author of more than 100 conference and journal papers mainly in the field of optical networking and networking performance evaluation. 